UAHEP-933

April 1993

\title{
DEFORMED WONG PARTICLES
}

\author{
A. Stern and I. Yakushin \\ Department of Physics, University of Alabama, \\ Tuscaloosa, AL 35487, USA.
}

\begin{abstract}
By generalizing the Feynman proof of the Lorentz force law, recently reported by Dyson, we derive equations of motion for particles possessing internal degrees of freedom $I^{a}$ which do not, in general, generate a finite algebra. We obtain consistency criteria for the fields which interact with such particles. It is argued that when a particle with internal $S U_{q}(2)$ degrees of freedom is coupled to $S U(2)$ gauge fields, $S U(2)$ gauge invariance is broken to $U(1)$. We further claim that when such an $S U_{q}(2)$ particle acts as a source for the field theory, the second rank antisymmetric field tensor, in general, cannot be globally defined.
\end{abstract}




\section{Introduction}

Recently there has been interest in constructing gauge theories based on quantum algebras[任]. Among the motivations for this activity is the hope of introducing a new symmetry breaking mechanism in gauge theories which could eventually be used to generate masses for vector fields. In the previous approaches, the Lie algebras associated with gauge fields were deformed, with the resulting field components having nontrivial commutation properties. In this article, we shall rather be interested in deforming the Lie algebras associated with particles which can couple to gauge fields. For us, the field components are c-numbers at the classical level. More generally, we shall examine the dynamics of a particle possessing internal degrees of freedom $I^{a}$ that do not generate a finite algebra. To obtain the dynamics, we need only postulate the particle's Poisson brackets and assume the existence of a Hamiltonian evolution. The procedure is completely analogous to the Feynman proof of the Lorentz force law, recently reported by Dyson[2]. The proof has been generalized to the case of a particle interacting with gravity and Yang-Mills fields in ref. [3], and a particle with external (or spin) $S U_{q}(2)$ degrees interacting with a magnetic field in ref. [4]. Our results offer a further generalization. They can be applied to the case of a particle with internal (or isospin) $S U_{q}(2)$ degrees of freedom interacting with an $S U(2)$ gauge field. We argue that when this happens, $S U(2)$ gauge invariance is broken to $U(1)$. We later obtain consistency criteria for fields having such a particle as a source and claim that in this case second rank antisymmetric field tensors cannot, in general, be globally defined.

The outline of this article is as follows: We obtain the generalized Lorentz force law in Section 2. In Section 3, we apply it to the case where $I^{a}$ span a Lie algebra and in so doing recover the Wong particle equations [5]. The case where $I^{a}$ defines an $S U_{q}(2)$ algebra, and the breaking of $S U(2)$ gauge invariance is discussed in Section 4. Finally, 
the consistency criteria for the associated field equations is examined in Section 5 .

\section{The Generalized Particle Equations}

We begin by examining a classical nonrelativistic particle of mass $m$. The relativistic generalization will be considered later. We denote the spatial coordinates of the particle by $x^{i}$ and its velocity by $v^{i}, i=1,2,3$. They are functions of some time parameter $t$, and

$v^{i}=\dot{x}^{i}$, where the dot denotes time differentiation. For Poisson brackets involving $x$ and $v$, we postulate the following:

$$
\begin{aligned}
& \left\{x^{i}, x^{j}\right\}=0 \\
& \left\{x^{i}, v^{j}\right\}=\frac{1}{m} \delta^{i j} .
\end{aligned}
$$

Next, we introduce an internal degree of freedom which we denote by $I^{a}=I^{a}(t)$, $a=1, \ldots, D$, and assume the general Poisson bracket relations

$$
\begin{aligned}
& \left\{I^{a}, I^{b}\right\}=C^{a b}(I), \\
& \left\{x^{i}, I^{a}\right\}=0 .
\end{aligned}
$$

More generally, we assume that for arbitrary functions $A=A(x, I)$ and $B=B(x, I)$ we can write

$$
\{A, B\}=C^{a b}(I) \delta_{a} A \delta_{b} B
$$

in any local region of phase space, where $\delta_{a}$ denotes a derivative with respect to $I^{a}$. Here we shall make no special assumptions for the function $C^{a b}(I)$, except

$$
C^{a b}=-C^{b a} \quad \text { and } \quad \delta_{d} C^{b c} C^{a d}+\delta_{d} C^{c a} C^{b d}+\delta_{d} C^{a b} C^{c d}=0
$$

which follows from antisymmetry of the Poisson bracket and the Jacobi identity. Thus the I's need not generate a finite algebra. 
From the above Poisson bracket relations, along with the assumption that $\dot{v}^{i}$ and $\dot{I}^{a}$ are functions of $x, v, I$ and $t$ only, we can show that the equation of motion for the particle must be of the form

$$
\begin{aligned}
& m \dot{v}^{i}=\mathcal{F}^{i j}(x, t, I) v^{j}+\mathcal{F}^{i 0}(x, t, I), \\
& \dot{I}^{a}=-\mathcal{A}^{i a}(x, t, I) v^{i}-\mathcal{A}^{0 a}(x, t, I),
\end{aligned}
$$

where the "fields" $\mathcal{F}^{\mu \nu}(x, t, I)=-\mathcal{F}^{\nu \mu}(x, t, I)$ and "potentials" $\mathcal{A}^{\mu a}(x, t, I)[\mu \nu, \ldots=$ $0,1,2,3]$ satisfy the following consistency conditions:

$$
\begin{gathered}
\mathcal{D}^{\lambda} \mathcal{F}^{\mu \nu}+\mathcal{D}^{\mu} \mathcal{F}^{\nu \lambda}+\mathcal{D}^{\nu} \mathcal{F}^{\lambda \mu}=0, \\
\delta_{d} \mathcal{F}^{\mu \nu} C^{a d}=\mathcal{D}^{\mu} \mathcal{A}^{\nu a}-\mathcal{D}^{\nu} \mathcal{A}^{\mu a}, \\
\delta_{d} C^{a b} \mathcal{A}^{\mu d}=\delta_{d} \mathcal{A}^{\mu b} C^{a d}-\delta_{d} \mathcal{A}^{\mu a} C^{b d},
\end{gathered}
$$

We define $\mathcal{D}^{\mu}$ by

$$
\mathcal{D}^{\mu} \equiv \partial^{\mu}-\mathcal{A}^{\mu d} \delta_{d}
$$

$\partial^{j}$ and $\partial^{0}$ denoting partial differentiation with respect to $x_{j}$ and $t$, respectively.

Following Feynman[2], to prove these results we define $\mathcal{F}^{i j}$ and $\mathcal{A}^{i a}$ according to

$$
\mathcal{F}^{i j}=-\mathcal{F}^{j i} \equiv m^{2}\left\{v^{i}, v^{j}\right\} \quad \text { and } \quad \mathcal{A}^{i a} \equiv m\left\{v^{i}, I^{a}\right\}
$$

By applying the Jacobi identity involving $x^{i}, v^{j}$ and $v^{k}$, we find that $x^{i}$ has zero Poisson bracket with $\mathcal{F}^{j k}$, while from the Jacobi identity involving $x^{i}, v^{j}$ and $I^{a}$, we find that $x^{i}$ has zero Poisson bracket with $\mathcal{A}^{j a}$. It is for this reason that $\mathcal{F}^{i j}$ and $\mathcal{A}^{i a}$ can be functions of $x$ and $I$ only. The eq. (10) with index $\mu=i$ follows simply from the Jacobi identity involving $I^{a}, I^{b}$ and $v^{i}$. Additional Jacobi identities give the following consistency conditions on $C^{a b}, \mathcal{F}^{i j}$ and $\mathcal{A}^{i a}$ :

$$
\frac{1}{m} \delta_{d} \mathcal{F}^{i j} C^{a d}=\left\{v^{j}, \mathcal{A}^{i a}\right\}-\left\{v^{i}, \mathcal{A}^{j a}\right\}
$$




$$
\left\{v^{i}, \mathcal{F}^{j k}\right\}+\left\{v^{j}, \mathcal{F}^{k i}\right\}+\left\{v^{k}, \mathcal{F}^{i j}\right\}=0
$$

By taking the time derivative of the Poisson bracket relations, and assuming the usual Leibniz rule, we can deduce the general form for the equations of motion for the system. The time derivative of eqs. (2) and (4), leads to

$$
m^{2}\left\{x^{i}, \dot{v}^{j}\right\}=-\mathcal{F}^{i j}(x, t, I) \text { and } m\left\{x^{i}, \dot{I}^{a}\right\}=-\mathcal{A}^{i a}(x, t, I),
$$

respectively. Then from the Poisson brackets (1), (2) and (4), $\dot{v}^{i}$ and $\dot{I}^{a}$ must be of the form (6) and (7). We can obtain conditions on the function $\mathcal{A}^{0 a}$ by taking the time derivative of $(3)$, leading to

$$
\left\{\dot{I}^{a}, I^{b}\right\}-\left\{\dot{I}^{b}, I^{a}\right\}=\delta_{d} C^{a b} \dot{I}^{d}
$$

After substituting (7) and using (10) with index $\mu=i$, we find condition (10) with index $\mu=0$.

The remaining conditions are obtained by taking the time derivative of eqs. (11). By differentiating $\mathcal{A}^{i a}=m\left\{v^{i}, I^{a}\right\}$ with respect to $t$ and using (6) and (7), we get

$$
\begin{aligned}
& \partial^{0} \mathcal{A}^{i a}+\partial^{j} \mathcal{A}^{i a} v_{j}-\delta_{d} \mathcal{A}^{i a} \mathcal{A}^{j d} v_{j}-\delta_{d} \mathcal{A}^{i a} \mathcal{A}^{0 d} \\
= & \delta_{d} \mathcal{F}^{i j} C^{d a} v_{j}+\delta_{d} \mathcal{F}^{i 0} C^{d a}-m\left\{v^{i}, \mathcal{A}^{j a}\right\} v_{j}-m\left\{v^{i}, \mathcal{A}^{0 a}\right\} .
\end{aligned}
$$

We can equate terms linear in $v_{j}$ and terms independent of $v_{j}$, leading to the two separate conditions. One of them is

$$
\mathcal{D}^{i} \mathcal{A}^{j a}=\partial^{i} \mathcal{A}^{j a}-\delta_{d} \mathcal{A}^{j a} \mathcal{A}^{i d}=-m\left\{v^{i}, \mathcal{A}^{j a}\right\}
$$

where we have used (12). If we more generally assume that

$$
\mathcal{D}^{i} f(x, I)=-m\left\{v^{i}, f(x, I)\right\}
$$

for any function $f(x, I)$, then the other condition is just $(9)$ with $(\mu \nu)=(i 0)$. Also for this, we have used the result that $\left\{v^{i}, \mathcal{A}^{j a}\right\}$ and $\left\{v^{i}, \mathcal{A}^{0 a}\right\}$ are independent of $v$, which 
follows from the Jacobi identity involving $x, v, \mathcal{A}^{i a}$, and $x, v, \mathcal{A}^{0 a}$, respectively. Upon using (16), the condition (12) reduces to $(9)$ with $(\mu \nu)=(i j)$.

By differentiating $\mathcal{F}^{j i}=m^{2}\left\{v^{j}, v^{i}\right\}$ with respect to $t$ and using (6) and (7), we get

$$
\begin{aligned}
& \partial^{0} \mathcal{F}^{j i}+\partial^{k} \mathcal{F}^{j i} v_{k}-\delta_{d} \mathcal{F}^{j i} \mathcal{A}^{k d} v_{k}-\delta_{d} \mathcal{F}^{j i} \mathcal{A}^{0 d} \\
= & m\left(\left\{\mathcal{F}^{j k}, v^{i}\right\} v_{k}-\left\{\mathcal{F}^{i k}, v^{j}\right\} v_{k}+\left\{\mathcal{F}^{j 0}, v^{i}\right\}-\left\{\mathcal{F}^{i 0}, v^{j}\right\}\right) .
\end{aligned}
$$

Again we can equate terms linear in $v_{i}$ and terms independent of $v_{i}$, leading to the two separate conditions: (8) with $(\mu \nu \lambda)=(i j 0)$ and

$$
\frac{1}{m} \mathcal{D}^{k} \mathcal{F}^{j i}=\left\{v^{j}, \mathcal{F}^{i k}\right\}-\left\{v^{i}, \mathcal{F}^{j k}\right\}
$$

Substituting the latter into eq. (13) then gives $(8)$ with $(\mu \nu \lambda)=(i j k)$. We thereby have verified all equations (6-10).

In the above proof we have assumed the Leibniz rule for the time derivative acting on a Poisson bracket; that is,

$$
\frac{d}{d t}\{A, B\}=\{\dot{A}, B\}+\{A, \dot{B}\}
$$

This may not be valid in general[3]. However, it is true if the system admits a Hamiltonian $H$, and the equations of motion can be written as Hamilton's equations of motion using $H$. For our system, we can find a Hamiltonian. It is,

$$
H=\frac{m}{2} v^{i} v^{i}+H_{I}(x, I) .
$$

We can then write the equations of motion (6) and (7) (along with $\dot{x}^{j}=v^{j}$ ) according to:

$$
\dot{I}^{a}=\left\{I^{a}, H\right\}, \quad \dot{x}^{j}=\left\{x^{j}, H\right\} \quad \text { and } \quad \dot{v}^{j}=\left\{v^{j}, H\right\}+\frac{\partial v^{j}}{\partial t}
$$

if we assume (11) and the following Poisson brackets for the interaction Hamiltonian $H_{I}$ :

$$
m\left\{v^{j}, H_{I}\right\}=\mathcal{F}^{j 0}-m \frac{\partial v^{j}}{\partial t} \quad \text { and } \quad\left\{I^{a}, H_{I}\right\}=-\mathcal{A}^{0 a}
$$


Here we are allowing for an explicit time dependence in the velocities $v^{i}$. Later we shall give an expression for $v^{i}$ and $H_{I}$ in terms of canonical phase space variables.

It is easy to generalize this system to the relativistic case. For this we can keep all the Poisson brackets (1-4), as well as (11) and (20), while we replace the Hamiltonian (18) by

$$
H=m \sqrt{1+v^{i} v^{i}}+H_{I}(x, I) .
$$

Now $\dot{x}^{j} \neq v^{j}$; Rather, we have $\dot{x}^{j}=v^{j} / \sqrt{v^{i} v^{i}+1}$. This is obtained from the Hamilton's equations of motion (19), along with

$$
\begin{gathered}
\frac{d}{d t}\left(\frac{m \dot{x}^{\mu}}{\sqrt{-\dot{x}^{\rho} \dot{x}_{\rho}}}\right)=\mathcal{F}^{\mu \nu}(x, I) \dot{x}_{\nu}, \\
\dot{I}^{a}=-\mathcal{A}^{\mu a}(x, I) \dot{x}_{\mu},
\end{gathered}
$$

where $x_{\mu}$ are components of a four-vector with $x_{0}=t$ and we use the Minkowski metric tensor $\left[\eta_{\mu \nu}\right]=\operatorname{diag}(-1,1,1,1)$.

As defined so far, the above theory resembles a Kaluza-Klein theory because the "fields" $\mathcal{F}^{\mu \nu}$ and "potentials" $\mathcal{A}_{\mu}^{a}$ are functions of internal coordinates $I$, as well as spacetime coordinates $x$. To reduce the theory to one which is defined on four dimensional space- time, it is necessary to make certain assumptions on the fields, such as they factorize into space-time dependent and internal space dependent pieces. Ansätze for the fields must be consistent with the conditions (8-10). We shall also require that the ansätze do not put restrictions on the particle degrees of freedom.

Now define $\mathcal{A}^{a}=\mathcal{A}^{a}(x, I)$ to be the one-form on Minkowski space, with components $\mathcal{A}^{\mu a}$. For $\mathcal{A}^{a}$ we choose the following:

$$
\mathcal{A}^{a}(x, I)=g C^{a b}(I) A_{b}(x),
$$

where $g$ is a constant and $A_{b}$ is a one-form on space-time. Eq. (24) satisfies eq. (10) for all values of $x$ and $I$, and for any $C^{a b}(I)$ fulfilling eq. (5). Upon substituting the ansatz 
(24) into eq. (9), we get

$$
C^{a b}(I)\left(\frac{1}{g} \delta_{b} \mathcal{F}(x, I)-d A_{b}(x)-\frac{g}{2} \delta_{b} C^{d e}(I) A_{d}(x) \wedge A_{e}(x)\right)=0,
$$

$\mathcal{F}$ being the two-form on Minkowski space, with components $\mathcal{F}^{\mu \nu} . d$ and $\wedge$ denote exterior derivative on Minkowski space and exterior product, respectively. Ignoring $I$ independent terms, eq. (25) is solved by

$$
\frac{1}{g} \mathcal{F}(x, I)=d A_{a} I^{a}+\frac{g}{2} A_{a} \wedge A_{b} C^{a b}(I) .
$$

With $\mathcal{A}^{a}$ and $\mathcal{F}$ given in eqs. (24) and (26), eq. (8) follows as an identity. [More generally, we may add an $I$ independent two-form to (26), and from (8) that two-form is closed.]

Starting from the ansatz (24) it is now easy to find an explicit form for the velocities $v^{i}$ and the interaction Hamiltonian $H_{I}$ appearing in eq. (18) [or (21)] in terms of canonical coordinates and momenta. We denote the canonical momenta by $p^{i}$ and assume that it has zero Poisson brackets with the internal variables. Thus, $\left\{p^{i}, p^{j}\right\}=\left\{p^{i}, I^{a}\right\}=$ 0 and $\left\{x^{i}, p^{j}\right\}=\delta^{i j}$. In terms of $x^{i}, p^{i}$ and $I^{a}$, we can define $v^{i}$ and $H_{I}$ by:

$$
m v_{i}(x, p, I)=p_{i}-g A_{a}^{i}(x) I^{a} \quad \text { and } \quad H_{I}(x, I)=-g A_{a}^{0}(x) I^{a} .
$$

From these definitions we then recover eqs. (11) and (20). The resulting Hamiltonian (18) [or (21)] is identical to that of a Wong particle [5].

\section{The Wong Equations}

Yang-Mills theory is recovered when $C^{a b}$ are linear functions of $I$, the coefficients being the structure constants associated with some Lie algebra $G$. That is,

$$
C^{a b}(I)=c^{a b}{ }_{d} I^{d}
$$

with eqs. (5) corresponding to $c^{a b}{ }_{d}=-c^{b a}{ }_{d}$ and $\quad c^{b c}{ }_{d} c^{a d}{ }_{e}+c^{c a}{ }_{d} c^{b d}{ }_{e}+c^{a b}{ }_{d} c^{c d}{ }_{e}=0$. Then from eqs. (24) and (26), $\mathcal{A}^{a}$ and $\mathcal{F}$ are also linear functions of $I, A_{b}=A_{b}(x)$ 
corresponding to Yang-Mills connection one-forms and $g$ being the coupling constant. If we write

$$
\mathcal{F}(x, I)=g F_{d}(x) I^{d}
$$

then we can identify $F_{d}=F_{d}(x)$ with the field strength two-form for Yang-Mills theory,

$$
F_{d}=d A_{d}+\frac{g}{2} c^{b e}{ }_{d} A_{b} \wedge A_{e}
$$

from which it follows that (8) is the usual Bianchi identity for Yang-Mills fields,

$$
d F_{a}+g c^{b d} A_{b} \wedge F_{d}=0
$$

Eqs. (22) and (23) correspond to the Wong equations of motion for a particle in a nonabelian gauge field [5].

\section{The Q-Deformed Wong Equations}

For general functions $C^{a b}(I)$ of $I, \mathcal{F}(x, I)$ does not factorize as in eq. (29). In the case of quantum algebras, some components $C^{a b}(I)$ are linear functions of $I^{a}$, while others are nonlinear in $I^{a}$. We next consider the example of $S U_{q}(2)$.

The $S U_{q}(2)$ algebra is standardly realized for quantum operators $\mathbf{I}^{+}, \mathbf{I}^{-}$and $\mathbf{I}^{0}$ by the commutation relations:

$$
\left[\mathbf{I}^{0}, \mathbf{I}^{ \pm}\right]= \pm \mathbf{I}^{ \pm}, \quad\left[\mathbf{I}^{+}, \mathbf{I}^{-}\right]=\left[2 \mathbf{I}^{0}\right]_{q} \equiv \frac{q^{2 \mathbf{I}^{0}}-q^{-2 \mathbf{I}^{0}}}{q-q^{-1}}
$$

(These commutation relations reduce to the $S U(2)$ algebra relations in the limit $q \rightarrow 1$.) To obtain the corresponding classical system, let us replace the quantum operators $\mathbf{I}^{+}, \mathbf{I}^{-}$ and $\mathbf{I}^{0}$ by classically commuting variables which we denote by $I^{1}+i I^{2}, I^{1}-i I^{2}$ and $I^{3}$, respectively. We also replace the commutation relations (31) by $i$ times Poisson brackets. The result is:

$$
\left\{I^{2}, I^{3}\right\}=I^{1}, \quad\left\{I^{3}, I^{1}\right\}=I^{2} \quad \text { and } \quad\left\{I^{1}, I^{2}\right\}=\frac{1}{2}\left[2 I^{3}\right]_{q} .
$$


From these relations, we identify the functions $C^{a b}$ according to: $C^{23}=I^{1}, C^{31}=$ $I^{2}$ and $C^{12}=\frac{1}{2}\left[2 I^{3}\right]_{q}$. Then we can write

$$
\begin{gathered}
\mathcal{A}^{a}(x, I)=g \epsilon^{a b c} A_{b}(x)\left(I^{c}+\delta^{c 3} h\left(I^{3}\right)\right), \\
\mathcal{F}(x, I)=g F_{a}(x) I^{a}+g^{2} A_{1} \wedge A_{2} h\left(I^{3}\right), \\
\\
h\left(I^{3}\right)=\frac{1}{2}\left[2 I^{3}\right]_{q}-I^{3},
\end{gathered}
$$

where $F_{a}$ is the $S U(2)$ Yang-Mills two-form eq. (30) with $c^{a b}{ }_{d}=\epsilon^{a b d}$. From eq. (8) we again get identities involving the fields.

Although we have not deformed the Wong particle Hamiltonian (18) [or (21)], we have deformed the Poisson brackets (32) from the $S U(2)$ case, and consequently also the equations of motion from the $S U(2)$ case. We thereby obtain "q-deformed" or $S U_{q}(2)$ Wong equations (22) and (23), with $\mathcal{A}^{a}$ and $\mathcal{F}$ defined in (33) and (34). From them we can show that

$$
\left(I^{1}\right)^{2}+\left(I^{2}\right)^{2}+\frac{1}{2 \ln q}\left(\frac{q^{2 I^{3}}+q^{-2 I^{3}}}{q-q^{-1}}-\frac{1}{\ln q}\right)
$$

is a constant of the motion. The term $\frac{1}{\ln q}$ was subtracted in parenthesis so that (35) converges in the limit of $q \rightarrow 1$. In that limit, it just becomes the classical analogue of the quadratic Casimir operator for $S U(2)$. It is not hard to show that (35) has zero Poisson bracket with $I^{a}$, and consequently all phase space variables, for any value of $q$. Hence, it is the classical analogue of a Casimir operator for $S U_{q}(2)$.

For $S U_{q}(2), \mathcal{F}(x, I)$ given in eq. (34) is invariant under infinitesimal $U(1)$ gauge transformations. Under such transformations, $A_{a}$ and $I^{a}$ undergo the infinitesimal changes:

$$
\delta A_{a}=\delta_{a 3} d \Lambda_{3}+g \epsilon_{a b 3} A_{b} \Lambda_{3} \quad, \quad \delta I^{a}=g \epsilon_{a b 3} I^{b} \Lambda_{3},
$$

where $\Lambda_{3}$ is an infinitesimal function of the particle space-time coordinates. It follows that the $S U_{q}(2)$ Wong equation (22) is invariant under $U(1)$ gauge transformations. The same is true for the equation of motion (23) using (33). 
If we were to interpret $A_{a}$ as connection one-forms for $S U(2)$ Yang-Mills theory, then eq. (36) corresponds to a $U(1)$ subgroup of $S U(2)$ Yang-Mills transformations. Under the full set of $S U(2)$ Yang-Mills transformations an infinitesimal change in $A_{a}$ is given by

$$
\delta A_{a}=d \Lambda_{a}+g \epsilon_{a b c} A_{b} \Lambda_{c}
$$

where $\Lambda_{a}$ are infinitesimal functions of the space-time coordinates. Is there a compensating transformation on $I^{a}$ such that the $S U_{q}(2)$ Wong equations are invariant for $\Lambda_{1}, \Lambda_{2} \neq 0$ ? We now argue that the answer is no. In order that $\mathcal{F}(x, I)$ given in eq. (34) be invariant under (37), $I^{a}$ must undergo a change $\delta I^{a}$, which satisfies the following equation:

$$
F_{b}\left(\delta I^{b}+g \epsilon_{a b c} I^{a} \Lambda_{c}\right)+g \epsilon_{a b 3} A_{a} \wedge\left(A_{b} f\left(I^{3}\right) \delta I^{3}+\left(d \Lambda_{b}+g \epsilon_{b c d} A_{c} \Lambda_{d}\right) h\left(I^{3}\right)\right)=0
$$

where $f\left(I^{3}\right)=\frac{1}{2} \frac{d h}{d I^{3}}$. But there exists no solution for $\delta I^{a}$ for arbitrary $A_{b}$, and arbitrary $\Lambda_{1}, \Lambda_{2}$. To see this, consider gauge transformations about the first axis, $\Lambda_{a}=\delta_{a 1} \Lambda_{1}$ and set $F_{2}=F_{3}=A_{1}=0$. Then for arbitrary $A_{2}$, the above condition states that

$$
A_{3} \delta I^{1}=d \Lambda_{1} h\left(I^{3}\right)
$$

But $A_{3}$ and $d \Lambda_{1}$ are independent (closed) one-forms on Minkowski space. Hence, the condition cannot be satisfied. The same conclusion is reached when we consider transformations about the second axis, $\Lambda_{a}=\delta_{a 2} \Lambda_{2}$ and set $F_{1}=F_{3}=A_{2}=0$. We therefore conclude that in the presence of $S U_{q}(2)$ Wong particles, the $S U(2)$ gauge invariance of $S U(2)$ Yang-Mills theory is broken to $U(1)$.

\section{The Field Equations}

In the above generalization of the Feynman proof of the Lorentz force equation, the dynamics of particles has been fully specified [eqs. (22) and (23)]. The same cannot be said about the dynamics of fields. Eqs. (8-10) are insufficient for determining the field 
dynamics. This, of course, was also the case for electromagnetism. The Gauss' law and Ampere's law actually did not follow in the proof of Feynman[2] and had to be postulated by hand. Not surprisingly then, we too must postulate additional field equations in order to fully specify the dynamics. In these equations, particles now act as sources for the fields. These equations are not completely arbitrary. They must satisfy certain consistency criteria when the particle sources possess internal degrees of freedom $I^{a}$. To see this, we introduce the space-time dependent quantities $J_{\mu}^{a}(y)$ and $\Sigma_{\mu}^{a b}(y)=-\Sigma_{\mu}^{b a}(y)$ and define them as follows:

$$
\begin{aligned}
J_{\mu}^{a}(y) & =\int d t \delta^{4}(y-x(t)) I^{a}(t) \dot{x}_{\mu}(t), \\
\Sigma_{\mu}^{a b}(y) & =\int d t \delta^{4}(y-x(t)) C^{a b}[I(t)] \dot{x}_{\mu}(t) .
\end{aligned}
$$

Here $x_{\mu}(t)$ and $I^{a}(t)$ are the space-time coordinates and internal coordinates, respectively, of the source particle and the integration is over the particle world line. Now by multiplying the particle equation $(23)$ by $\delta^{4}(y-x(t))$ and integrating over $t$, we obtain the following relations:

$$
\frac{\partial}{\partial y_{\mu}} J_{\mu}^{a}(y)+g A_{b}^{\mu}(y) \Sigma_{\mu}^{a b}(y)=0,
$$

where we have assumed (24). Now if we equate $J_{\mu}^{a}(y)$ and $\Sigma_{\mu}^{a b}(y)$ to some functionals of $A_{\mu}^{a}(y)$ (and possibly other fields) along with their derivatives, then eqs. (38) and (39) represent field equations for the system in the presence of a source. Furthermore, eq. (40) then corresponds to a set of consistency criteria which the fields must satisfy.

For the case where the source is a Wong particle, the "charges" $I^{a}$ and $C^{a b}[I]$ appearing in (38) and (39) are linearly related [eq. (28)]. Consequently, so are $\Sigma_{\mu}^{a b}$ and $J_{\mu}^{d}: \Sigma_{\mu}^{a b}=$ $c^{a b}{ }_{d}^{d}$. Here, eq. (40) can be written

$$
\left(D^{\nu} J_{\nu}\right)^{a}=0
$$

$D^{\nu}\left(\left[D^{\nu}\right]_{b}^{a}=\partial^{\nu} \delta_{b}^{a}+g c^{a d}{ }_{b} A_{d}^{\nu}\right)$ denoting the covariant derivative. This condition is just the statement that the Yang-Mills current is covariantly conserved. It is identically 
satisfied with the usual choice of Yang-Mills field equations,

$$
\left(D^{\nu} F_{\nu \mu}\right)^{a}=J_{\mu}^{a}
$$

since upon substituting into the condition (41), we get $D^{\mu}\left(D^{\nu} F_{\nu \mu}\right) \equiv 0$.

For the case where the source is a q-deformed Wong particle, the "charges" $C^{a b}[I]$ and $I^{a}$ are not all linearly related, and no simple relation exists between all the $\Sigma_{\mu}^{a b}$ and $J_{\mu}^{d}$. For $S U_{q}(2)$ particle sources, there are six quantities $\Sigma_{\mu}^{a b}$ and $J_{\mu}^{a}$, but from eqs. (32) we can make the identifications: $\Sigma_{\mu}^{23}=J_{\mu}^{1}$ and $\Sigma_{\mu}^{31}=J_{\mu}^{2}$. Four independent quantities remain which we denote by $J_{\mu}^{(q) a}$ and $\Delta_{\mu}$. We define them by:

$$
J_{\mu}^{(q) 1}=J_{\mu}^{1}, \quad J_{\mu}^{(q) 2}=J_{\mu}^{2}, \quad J_{\mu}^{(q) 3}=\Sigma_{\mu}^{12} \quad \text { and } \quad \Delta_{\mu}=\Sigma_{\mu}^{12}-J_{\mu}^{3} .
$$

From eqs. (38) and (39) we thus have four field equations. The conditions (40) can be written:

$$
\left(D^{\mu} J_{\mu}^{(q)}\right)^{a}=\partial^{\mu} \Delta_{\mu} \delta^{a 3}
$$

where $D^{\mu}$ is the covariant derivative for $S U(2)$. Using eqs. (38) and (39), we then have

$$
\left(D^{\mu} J_{\mu}^{(q)}\right)^{a}(y)=\delta^{a 3} \int d t \delta^{4}(y-x(t)) \frac{d}{d t} h\left(I^{3}(t)\right)
$$

In the limit $q \rightarrow 1, \Delta_{\mu}$ vanishes and $J_{\mu}^{(q) a} \rightarrow J_{\mu}^{a}$. Then eqs. (44) reduce to (41) and are solved by eq. (42). We thereby recover the field equations for $S U(2)$ gauge theory in that limit.

If we interpret $J_{\mu}^{(q) a}$ as the current associated with a q-deformed Wong particle, then eq. (44) shows that it is not covariantly conserved for arbitrary $q$ and $I^{a}$. [Here "covariantly" means with regard to $S U(2)$ transformations.] The same conclusion is reached upon taking $J_{\mu}^{a}$ to be the current associated with q-deformed particles. In terms of this current, the condition (40) becomes:

$$
\left(D^{\mu} J_{\mu}\right)^{a}=-g \epsilon^{a b 3} A_{b}^{\mu} \Delta_{\mu}
$$


Let us examine the case where $\frac{d h}{d t}$ is nonvanishing only for a finite segment $\mathbf{L}$ (begining at a time $t=t_{1}$ and ending at a time $\left.t=t_{2}\right)$ of an $S U_{q}(2)$ particle's world line. Now if in analogy to Yang-Mills theory, we were then to write

$$
\left(D^{\nu} F_{\nu \mu}\right)^{a}=J_{\mu}^{(q) a},
$$

we speculate that the fields components $F_{\mu \nu}^{a}(y)$ may not all be globally defined on $\mathbf{M} \backslash$ L, M denoting Minkowski space. To argue this point it is usefull to make the simplifying assumption that all fields and potentials in the 1 and 2 directions of internal space are zero. This could be done consistently if we were allowed to set $I^{1}=I^{2}=0$, for all $t$, and hence $J_{\mu}^{(q) 1}=J_{\mu}^{(q) 2}=0$. But with $I^{1}=I^{2}=0$ and $I^{3}$ changing, (35) cannot remain a constant of the motion. As an alternative, let us instead imagine that the vector $I$ precesses rapidly about the third axis. That is, $I^{1}$ and $I^{2}$ are oscillating rapidly (compared with $\left.I^{3}\right)$. In other words, $I^{1}$ and $I^{2}$ are "fast" variables, and $I^{3}$ is a "slow" variable. If we then time average over the "fast" variables we obtain the desired simplification; Namely, $J_{\mu}^{(q) 1}=J_{\mu}^{(q) 2}=0$. The resulting time averaged fields and potentials can then be made to point in the third direction in the internal space. Now define $* J^{(q) 3}$ to be a 3 -form whose components are dual to $J_{\mu}^{(q) 3}$. From eq. (44), $d * J^{(q) 3}$ is proportional to $\int d t \delta^{4}(y-x(t)) \frac{d}{d t} h\left(I^{3}(t)\right)$. If $S^{3}$ is a 3-sphere whose enclosing four dimensional volume $V^{4}$ contains $\mathbf{L}$, then from Stoke's theorem

$$
\int_{S^{3}} * J^{(q) 3}=\int_{V^{4}} d * J^{(q) 3} \propto \int_{\mathbf{L}} d t \frac{d}{d t} h\left(I^{3}(t)\right)=h\left(I^{3}\left(t_{2}\right)\right)-h\left(I^{3}\left(t_{1}\right)\right) .
$$

It follows that if $h\left(I^{3}\right)$ undergoes a nonzero change along $\mathbf{L}$, then $* J^{(q) 3}$ is a closed but not exact 3-form on $S^{3}$. Thus if we write $* J^{(q) 3}=d * F^{3}$, the two-form $* F^{3}$ (and hence the antisymmetric field tensor $F_{\mu \nu}^{3}$ ) cannot be defined everywhere on $S^{3}$. [More generally, if we define $* \Delta$ to be the 3 -form whose components are dual to $\Delta_{\mu}$ and if $h\left(I^{3}\right)$ undergoes a nonzero change along $\mathbf{L}$, then $* \Delta$ is closed but not exact on $S^{3}$. Note that for this to be valid no time averaging of the fields is necessary.] 
The above is an adaptation of a result found long ago by Rasetti and Regge 6 in the context of superfluid helium. There the analogue of $F_{\mu \nu}^{3}$ was the antisymmetric potential used to describe phonon excitations, while the source was interpreted as corresponding to the injection of helium atoms in the superfluid.

\section{Acknowledgements}

We are grateful to M. Lukin for discussions. We have been supported during the course of this work by the Department of Energy, USA under contract number DE-FG05-84ER-40141.

\section{References}

[1] I. Y. Aref'eva and I. V. Volovich, Mod. Phys. Lett. A6 (1991) 893; A. P. Isaev and Z. Popowicz, Phys. Lett B281 (1992) 271; Dubna preprint JINR E2-93-54; L. Castellani, Phys. Lett. B292 (1992) 93; K. Wu and R. J. Zhang, Comm. Theor. Phys. 17 (1992) 175; D. Bernard, Suppl. Prog. Theor. Phys. 102 (1992) 49; M. Hirayama, Prog. Theor. Phys. 88 (1992) 111.

[2] F. J. Dyson, Am. J. Phys. 58 (1990) 209.

[3] S. Tanimura, Ann. Phys. 220 (1992) 229.

[4] S. K. Soni, J. Phys. A25 (1992) L837.

[5] S. K. Wong, Nouvo Cim. A 65 (1970) 689; A. P. Balachandran, S. Borchardt and A. Stern, Phys. Rev. D17 (1978) 3247; A. P. Balachandran, G. Marmo, B.-S. Skagerstam and A. Stern, Gauge Symmetries and Fibre Bundles, Lecture Notes in Physics 188 (Springer-Verlag, Berlin, 1982). 
[6] M. Rasetti and T. Regge, Physica 80A (1975) 217; F. Lund and T. Regge, Phys. Rev. D14 (1976) 1524; A. P. Balachandran, V P. Nair, B.-S. Skagerstam and C. G. Trahern, Phys. Rev. D15 (1982) 1443. 